\title{
Novel Prognostic Score for Recurrent or Metastatic Head and Neck Cancer Patients treated with Nivolumab: Japanese Multi-Institutional Cohort Study
}

Kiyoshi Minohara

Nagoya City University

Takuma Matoba

Nagoya City University

Daisuke Kawakita ( $\nabla$ dk200811@gmail.com)

Nagoya City University

Gaku Takano

Nagoya City University

Keisuke Oguri

Konan Kosei Hospital

Akihiro Murashima

Nagoya City University

Kazuhiro Nakai

Nagoya City University

Sho Iwaki

Nagoya City University

Ayumu Hojo

Nagoya City University

Ayano Matsumura

Nagoya City University

Shinya Ozaki

Konan Kosei Hospital

Taijiro Ozawa

Toyohashi Municipal Hospital

Ikuma Harata

Kainan Hospital

Nobukazu Tanaka

Toyota Kosei Hospital

Shinichiro Maseki

Toyota Kosei Hospital

Hiroshi Tsuge

Anjo Kosei Hospital

Sae Imaizumi

Anjo Kosei Hospital

Shoji Mitsuya

Anjo Kosei Hospital

Kazuho Moribe

Ichinomiya Municipal City Hospital

Shinichi Esaki

Nagoya City University

Shinichi Iwasaki

Nagoya City University

Research Article

Keywords: Prognostic, RM-HNC, nivolumab therapy

Posted Date: June 3rd, 2021

DOI: https://doi.org/10.21203/rs.3.rs-576295/v1

License: (c) (i) This work is licensed under a Creative Commons Attribution 4.0 International License. Read Full License 
Version of Record: A version of this preprint was published at Scientific Reports on August 20th, 2021. See the published version at https://doi.org/10.1038/s41598-021-96538-7. 


\section{Abstract}

Although several prognostic factors in nivolumab therapy have been reported in patients with recurrent or metastatic head and neck cancer (RM-HNC), these factors remain controversial. Here, we conducted a multicenter retrospective cohort study to investigate the impact of clinico-hematological factors on survival in RM-HNC patients treated with nivolumab. We retrospectively reviewed $126 \mathrm{RM}$-HNC patients from seven institutes. We evaluated the prognostic effects of clinico-hematological factors on survival using Cox proportional hazard models. The median overall survival (OS) was 12.3 months, and the 1 year-OS rate was $51.2 \%$. Patients without immune-related adverse events, lower relative eosinophil count, worse best overall response, higher performance status, and higher modified Glasgow Prognostic Score had significantly worse survival rates. The prognostic score, generated by combining these significantly worse prognostic factors, was more closely associated with prognosis than each factor ( $p$ for trend<0.001). Our novel prognostic score utilizing clinicohematological factors might be useful to establish an individual treatment strategy in patients with RM-HNC treated with nivolumab therapy.

\section{Introduction}

Immune checkpoint inhibitors (ICls) have become standard therapy for various types of cancer ${ }^{1-4}$. Nivolumab, a programmed cell death 1 (PD-1) inhibitor, was approved in 2017 to treat platinum-refractory recurrent or metastatic head and neck cancer (RM-HNC) in Japan, receiving the results of CheckMate 141 trial ${ }^{1}$. Although nivolumab is highly effective in some patients, more than half of these patients do not have clinical benefits.

To date, several studies have reported the association of patient-related factors, including the best overall response (BOR), the occurrence of immune-related adverse events (irAEs), programmed death-ligand 1 (PD-L1) expression, high mutational burden, hematological inflammatory, and nutritional markers, and clinical outcomes in RM-HNC patients treated with nivolumab therapy ${ }^{5-13}$. However, the optimal prognostic factors for nivolumab therapy in patients with RM$\mathrm{HNC}$ are controversial. Furthermore, in considering the cost-effectiveness of ICl therapy, it is essential to elucidate the optimal prognostic factors in RM-HNC patients treated with ICl therapy.

Here, we conducted a multicenter retrospective cohort study to investigate the impact of clinico-hematological factors on survival in RM-HNC patients treated with nivolumab therapy among the Japanese population.

\section{Results}

\section{Patient Characteristics}

The median follow-up interval was 7.5 months (range, 0.5-33 months) for all patients in this study. The patient characteristics are summarized in Table 1. The median age was 68 years (range, 35-90 years), and men were predominant. The primary tumor sites were oral cavity cancer in 12 , oropharyngeal cancer in 27 , hypopharyngeal cancer in 20 , laryngeal cancer in 21 , sinonasal cancer in 13 , nasopharyngeal cancer in 11 , salivary gland cancer in 8 , external ear canal cancer in 7, and unknown primary cancer in 7. Fifty-four patients had locoregional diseases, and 72 patients had distant metastases. Regarding Eastern Cooperative Oncology Group Performance Status (ECOG PS), most patients were less than 1.

\section{IrAE profile}

The irAE profiles are shown in Supplementary Table S1. Forty-one patients (32.5\%) presented with 50 irAEs. The most common irAE category was endocrine irAEs (hypothyroidism, hypophysitis, etc.), followed by skin irAEs (rash, dermatitis, rash acneiform, etc.). There were 24 grade 3 or higher irAEs, and steroid therapy was administered to 25 patients.

\section{Clinical outcomes}

For all the patients, the median overall survival (OS) was 12.3 months (95\%Cl: 9.7-16.0), and 1 year-OS rate was 51.2\% (95\% Cl: 40.0-61.2), and the median progression-free survival (PFS) was 3.9 months $₫ 95 \% \mathrm{Cl}$ : $2.8-5.4 \rrbracket$ and 1 year-PFS rate was $14.6 \%$ (95\% Cl: 8.7-22.0) (Supplementary Fig S1). BOR to nivolumab therapy was complete response (CR) in 5 patients (4.0\%), partial response (PR) in 24 patients (19.0\%), stable disease (SD) in 48 patients (38.1\%), and progressive disease (PD) in 49 patients (38.9\%). After PD in nivolumab therapy, chemotherapy was administered to 44 patients. Among these patients, paclitaxel and cetuximab therapy was the most common (47.7\%) (Supplementary Table S2). BOR to chemotherapy after nivolumab was CR in 1 patient (2.3\%), PR in 17 patients (38.6\%), SD in 6 patients (13.6\%), and PD in 19 patients (43.2\%).

The association between clinico-hematological factors and clinical outcomes is shown in Table 2. In univariate analysis, OS was better for patients with any irAEs (HR, 0.49; 95\% Cl, 0.27-0.87; p= 0.016), higher REC (HR: 0.51, 95\% Cl: 0.31-0.86, p=0.011), and better BOR (HR: 0.18, 95\%Cl: 0.08-0.41, p<0.001). Moreover, OS was worse in patients with higher ECOG PS (HR, 3.14; 95\% Cl, 1.77-5.57; $\mathrm{p}<0.001)$ and higher modified Glasgow Prognostic Score (mGPS) (HR: 2.40, $95 \% \mathrm{Cl}: 1.41-4.10, \mathrm{p}=0.001)$. Furthermore, PFS was better for patients with any irAEs (HR: $0.55,95 \% \mathrm{Cl}: 0.36-0.84, \mathrm{p}=0.006)$, higher relative eosinophil count (REC) (HR: $0.68,95 \% \mathrm{Cl}: 0.46-1.00, p=0.049$ ), and better BOR (HR: $0.19,95 \% \mathrm{Cl}: 0.11-0.33, \mathrm{p}<0.001)$. Additionally, PFS was worse for patients with higher ECOG PS (HR, 1.90; 95\%Cl, 1.18-3.06; $p=0.008)$ and higher mGPS (HR: 2.02, 95\% Cl: 1.34-3.03, $p<0.001)$.

In multivariate analysis, OS was better for patients with any irAEs (HR: 0.47, 95\% Cl: 0.26-0.86, $\mathrm{p}=0.015)$, higher REC (HR: 0.49, 95\%Cl: $0.29-0.83, \mathrm{p}=0.008)$, and better BOR (HR: 0.18, 95\% Cl: 0.07-0.42, p< 0.001). Besides, OS was worse in patients with higher ECOG PS ( $\mathrm{HR}, 2.94 ; 95 \% \mathrm{Cl}, 1.55-5.55 ; \mathrm{p}<0.001)$ and higher mGPS (HR: 2.51, 95\% Cl: 1.45-4.35, p< 0.001). Furthermore, PFS was better for patients with any irAEs (HR: 0.52, 95\%Cl: 0.34-0.80, p=0.003), higher REC (HR: $0.68,95 \% \mathrm{Cl}: 0.46-1.00, p=0.048)$, and better BOR (HR: $0.17,95 \% \mathrm{Cl}: 0.10-0.30, p<0.001)$. Additionally, PFS was worse for patients with higher ECOG PS $(\mathrm{HR}, 2.17 ; 95 \% \mathrm{Cl}: 1.30-3.63, p=0.003)$ and higher mGPS (HR: $2.37,95 \% \mathrm{Cl}: 1.55-3.63, p<0.001)$. 
Finally, we investigated the impact of prognostic score using the sum of numbers from worse prognostic factors that were statistically significant in clinicohematological factors, including without irAEs, lower REC, worse BOR, higher ECOG PS, and higher mGPS (Fig 1 and Table 2). We found that higher scores were associated with worse OS and PFS, with a significant trend ( $p$ for trend $<0.001$ ), and were likely to be superior to each factor.

\section{Discussion}

This study found the prognostic effect of clinico-hematological factors, including the occurrence of irAEs, REC, BOR, ECOG PS, and mGPS in RM-HNC patients treated with nivolumab therapy. Furthermore, our analysis showed that the sum of numbers from worse prognostic factors in clinico-hematological factors might be the optimal prognostic score in the Japanese population.

Similar to previous studies reported ${ }^{5,14}$, we found that the occurrence of irAEs was significantly associated with better prognosis in RM-HNC patients treated with nivolumab therapy. Matsuo et al. reported that gastrointestinal irAEs were significantly associated with better PFS ${ }^{5}$. In other types of cancer, cutaneous, gastrointestinal, and endocrine irAEs are associated with better survival ${ }^{15,16}$. Although the endocrine irAEs category was most common in this study, we could not detect which type of irAE was strongly associated with clinical outcomes. However, since appropriate management of irAEs could lead to the clinical benefit of nivolumab therapy, early detection of irAEs and management in multidisciplinary teams should be advocated.

Additionally, we found that a better BOR to nivolumab therapy was associated with better survival. In the CheckMate 141 trial, the response rate in the nivolumab group was higher than that in the investigator's choice group, and tumor reduction was more durable with nivolumab ${ }^{1}$. Matsuki et al. also demonstrated that a better BOR was significantly associated with better survival ${ }^{17}$. It is obvious that response evaluation is important even when using immunotherapeutic agents.

Consistent with our results, Nishikawa et al. demonstrated that higher eosinophil counts and increases were associated with better survival ${ }^{13,18}$. Furthermore, eosinophil accumulation was associated with better survival in patients with melanoma treated with $\mathrm{ICI}^{19}$. They mentioned that the high number of peripheral blood eosinophils might reflect the high number of tumor-infiltrating eosinophils and the increase in tumor antigens due to tumor necrosis and collapse.

In this study, modified GPS and ECOG PS were associated with clinical outcomes in RM-HNC patients treated with nivolumab therapy. These factors are known to be prognostic factors in patients with $\mathrm{ICl}$ and other treatments ${ }^{20,21}$. Therefore, these biomarkers may be useful in various treatment modalities. The advantage of these biomarkers and eosinophil count, described above, is that they can be evaluated before nivolumab treatment. Since these two factors indicate the patient's general condition and/or inflammation, it might be better to use ICl without any symptoms associated with RM-HNC.

We demonstrated that the sum of numbers from worse prognostic factors could be the optimal prognostic score in RM-HNC patients treated with nivolumab. To the best of our knowledge, this is the first report to evaluate the impact of prognostic score associated with clinico-hematological factors, which are routinely available in clinical settings, on clinical outcomes in RM-HNC patients with nivolumab. However, the concern is that the poor nutritional condition and infection could affect these factors. Therefore, supportive therapy, including nutritional support, oral care, and smoking cessation, should be considered in patients with RM-HNC.

Regarding the primary tumor site, while we performed nivolumab therapy in patients not included in the CheckMate 141 trial, such as nasopharyngeal cancer, clinical outcomes were comparable to those included in the CheckMate 141 trial ${ }^{1,7}$. In patients excluded from the CheckMate 141 trial, the efficacy of nivolumab therapy has been reported with primary tumors at other sites, including the nasopharynx, while its efficacy is limited in the salivary gland cancer ${ }^{5,22-24}$. However, there is insufficient evidence for the optimal systematic therapy for HNC other than squamous cell carcinoma (SCC). A larger collaborative study to evaluate the efficacy of nivolumab therapy in patients with HNC other than SCC is required.

Regarding salvage chemotherapy following nivolumab therapy, we mainly performed paclitaxel and cetuximab therapy, and our clinical outcomes, including $41 \%$ ORR, were comparable to those of previous studies ${ }^{25-27}$. As mentioned above, since the efficacy of cetuximab and other chemotherapies following ICI has been reported, relatively early changes in treatment modalities might be acceptable.

Our study had several strengths. Since this is a multi-institutional cohort study, our sample size is one of the largest studies in patients with RM-HNC in a realworld setting. Second, detailed individual data were available for this study. Third, because physicians had no information on the association of clinichematological factors with survival, information bias appears unlikely. Fourth, we could perform the analysis adjusted for potential confounders, including detailed clinical information.

Moreover, several limitations should be mentioned. First, this study was conducted with a retrospective and multi-institutional design. Second, our information, especially hematological factors, reflected pre-treatment status only and not post-treatment factors, which might be associated with clinical outcomes. Third, we could not fully remove the potential effect of factors of infectious diseases, inflammation other than that derived from RM-HNC, and the use of glucocorticoid hormones.

In conclusion, we found that the occurrence of irAEs, higher REC, better BOR, lower ECOG PS, and lower mGPS were the better prognostic factors for survival in patients with RM-HNC treated with nivolumab. Furthermore, the sum of the numbers of worse prognostic factors might be the optimal prognostic score. Using this novel prognostic score, more effective treatment strategies, including nivolumab therapy, could be established for patients with RM-HNC.

\section{Materials And Methods}




\section{Patients}

We conducted a retrospective cohort study to investigate the efficacy and safety of nivolumab therapy in RM-HNC patients in seven institutes, including Nagoya City University Hospital, Konan Kosei Hospital, Toyohashi Municipal Hospital, Kainan Hospital, Toyota Kosei Hospital, Anjo Kosei Hospital, and Ichinomiya Municipal Hospital. Among these institutes, 126 RM-HNC patients were treated with nivolumab between April 2017 and November 2019 . The Institutional Review Board (IRB) of Nagoya City University Graduate School of Medical Sciences approved our protocols (approval number: 60-21-0001). Concerning consent to participate, patients could reject participation by opting out, to an announcement on Nagoya City University Hospital's website. This study was conducted in accordance with the principles of the Declaration of Helsinki.

\section{Treatment and follow-up}

All patients had been treated with platinum-based therapy before nivolumab therapy. Nivolumab was administered at a dose of $3 \mathrm{mg} / \mathrm{kg}$ every 2 weeks. The response to nivolumab therapy was evaluated according to Response Evaluation Criteria in Solid Tumor (RECIST) criteria version $1.1^{28}$, using computed tomography (CT) or magnetic resonance imaging (MRI) every 8 to 12 weeks. Patients in whom nivolumab administration was terminated due to clinically obvious disease progression were diagnosed with PD, even when image evaluation was not performed. Chemotherapy with cytotoxic agents was administered to patients diagnosed with PD. Follow-up was continued until death or the cut-off date (May 6, 2020).

\section{Prognostic effect of clinical and hematological factors}

This study evaluated the prognostic effect of clinical factors, including age, sex, primary tumor site, ECOG PS, site of recurrence, and platinum sensitivity. A platinum-refractory tumor was defined as a tumor that progressed within 6 months after the last platinum-based chemotherapy or a residual tumor after platinum-based chemoradiotherapy. A platinum-sensitive tumor was defined as a tumor that progressed from 6 months or longer after the last platinum-based chemotherapy. Moreover, we also evaluated the prognostic effect of hematological factors: REC, neutrophil to lymphocyte ratio (NLR), platelet to lymphocyte ratio (PLR), and mGPS. Hematological factors were calculated by eosinophil count, neutrophil count, lymphocyte count, platelet count, C-reactive protein (CRP), and albumin (Alb) in peripheral blood just before the start of nivolumab therapy. Regarding mGPS, patients with both elevated CRP level (>1.0 mg/dL) and decreased Alb ( $<3.5 \mathrm{~g} / \mathrm{dL})$ were assigned a score of 2 ; those with elevated CRP level $(>1.0 \mathrm{mg} / \mathrm{dL})$ and non-decreased Alb ( $\geq 3.5 \mathrm{~g} / \mathrm{dL})$ were assigned a score of 1 , and those with a non-elevated CRP level $(\leq 1.0 \mathrm{mg} / \mathrm{dL})$ were assigned a score of 0 according to a previous study ${ }^{29}$.

\section{Statistical analysis}

We investigated overall effectiveness, including BOR, PFS, and OS. BOR was defined as the best response from the initiation of nivolumab administration to PD. PFS was defined as the time from the first nivolumab administration to the date of PD or clinically unequivocal progression. OS was defined as the time from the first nivolumab administration to the date of death or the last visit. The irAEs were evaluated according to the protocol described in a previous study ${ }^{30}$. Toxicity was assessed using the Common Terminology Criteria for Adverse Events (CTCAE) version $5.0^{31}$.

Additionally, we evaluated the frequency of irAEs and the association between clinico-hematological factors and clinical outcomes in nivolumab therapy. The association of clinico-hematological factors with PFS or OS was assessed using the Kaplan-Meier product-limit method and univariate and multivariate Cox proportional hazards models. In the multivariate analysis, the forced entry method was performed. The measure of association in this study was hazard ratios (HRs) with 95\% confidence intervals (Cls). Statistical significance was set at $P<0.05$. Statistical analyses were performed using GraphPad Prism (version 9.00; GraphPad Software, San Diego, CA, USA) and EZR version 1.40 (Saitama Medical Center, Jichi Medical University, Saitama, Japan), which is a graphical user interface for R (R version 3.6.1, The R Foundation for Statistical Computing, Vienna, Austria) ${ }^{32}$. More precisely, it is a modified version of R commander designed to add statistical functions frequently used in biostatistics.

\section{Declarations}

\section{Ethical approval}

The study protocol was approved by the Institutional Review Board (IRB) of Nagoya City University Graduate School of Medical Sciences (\# 60-21-0001). Concerning consent to participate, patients could reject participation by opting out, to an announcement on Nagoya City University Hospital's website. Therefore, written informed consent was waived, which was approved by the IRB of Nagoya City University Graduate School of Medical Sciences. This study was conducted in accordance with the principles of the Declaration of Helsinki.

\section{Data availability}

The datasets generated in this study are available from the corresponding author on request.

\section{Acknowledgment}

The authors thank all the patients, their families, and caregivers. The authors thank all the clinicians for their involvement and contribution to the study. This work was supported by JSPS Grant-in-Aid for Young Scientists (B) to D. Kawakita (No. 17K18006) and T. Matoba (No. 19K18779), and by a JSPS Grant-in-Aid for Scientific Research (C) to D. Kawakita (No. 20K10508).

\section{Author contributions}


Study concept and design: K.M., T.M., D.K., and S.I.; acquisition of data: K.M., T.M., D.K., G.T., K.O., A.M., K.N., S.I., A.H., A.M., S.O., T.O., I.H., N.T., S.M., H.T., S.I., S.M., K.M., S.E., and S.I.; analysis and interpretation of data: K.M., T.M., and D.K.; statistical analysis: K.M., T.M., and D.K.; drafting of the manuscript: K.M., T.M., D.K., and S.I.; manuscript review: all authors.

\section{Conflict of interest}

All authors declare no conflict of interest associated with this study.

\section{References}

1. Ferris, R. L. et al. Nivolumab for Recurrent Squamous-Cell Carcinoma of the Head and Neck. N Engl J Med 375, 1856-1867, doi:10.1056/NEJMoa1602252 (2016).

2. Wolchok, J. D. et al. Overall Survival with Combined Nivolumab and Ipilimumab in Advanced Melanoma. N Engl J Med 377, 1345-1356, doi:10.1056/NEJMoa1709684 (2017).

3. Overman, M. J. et al. Durable Clinical Benefit With Nivolumab Plus Ipilimumab in DNA Mismatch Repair-Deficient/Microsatellite Instability-High Metastatic Colorectal Cancer. J Clin Onco/ 36, 773-779, doi:10.1200/jco.2017.76.9901 (2018).

4. Garon, E. B. et al. Pembrolizumab for the treatment of non-small-cell lung cancer. N Engl J Med 372, 2018-2028, doi:10.1056/NEJMoa1501824 (2015).

5. Matsuo, M. et al. Relationship between immune-related adverse events and the long-term outcomes in recurrent/metastatic head and neck squamous cell carcinoma treated with nivolumab. Oral Oncol 101, 104525, doi:10.1016/j.oraloncology.2019.104525 (2020).

6. Hanai, N. et al. Effectiveness and safety of nivolumab in patients with head and neck cancer in Japanese real-world clinical practice: a multicenter retrospective clinical study. Int J Clin Oncol 26, 494-506, doi:10.1007/s10147-020-01829-0 (2021).

7. Ferris, R. L. et al. Nivolumab vs investigator's choice in recurrent or metastatic squamous cell carcinoma of the head and neck: 2-year long-term survival update of CheckMate 141 with analyses by tumor PD-L1 expression. Oral Onco/ 81, 45-51, doi:10.1016/j.oraloncology.2018.04.008 (2018).

8. Yarchoan, M., Hopkins, A. \& Jaffee, E. M. Tumor Mutational Burden and Response Rate to PD-1 Inhibition. N Engl J Med 377, 2500-2501, doi:10.1056/NEJMc1713444 (2017).

9. Hanna, G. J. et al. Frameshift events predict anti-PD-1/L1 response in head and neck cancer. JCl Insight 3, doi:10.1172/jci.insight.98811 (2018).

10. Yu, Y. et al. Pretreatment neutrophil to lymphocyte ratio in determining the prognosis of head and neck cancer: a meta-analysis. BMC Cancer 18,383 , doi:10.1186/s12885-018-4230-z (2018).

11. Proctor, M. J. et al. An inflammation-based prognostic score (mGPS) predicts cancer survival independent of tumour site: a Glasgow Inflammation Outcome Study. Br J Cancer 104, 726-734, doi:10.1038/sj.bjc.6606087 (2011).

12. Matsuki, T. et al. Hematological predictive markers for recurrent or metastatic squamous cell carcinomas of the head and neck treated with nivolumab: $A$ multicenter study of 88 patients. Cancer Med 9, 5015-5024, doi:10.1002/cam4.3124 (2020).

13. Nishikawa, D. et al. Eosinophil prognostic scores for patients with head and neck squamous cell carcinoma treated with nivolumab. Cancer Sci 112, 339346, doi:10.1111/cas.14706 (2021)

14. Okamoto, I. et al. Efficacy and safety of nivolumab in 100 patients with recurrent or metastatic head and neck cancer - a retrospective multicentre study. Acta Otolaryngol 139, 918-925, doi:10.1080/00016489.2019.1648867 (2019).

15. Freeman-Keller, M. et al. Nivolumab in Resected and Unresectable Metastatic Melanoma: Characteristics of Immune-Related Adverse Events and Association with Outcomes. Clin Cancer Res 22, 886-894, doi:10.1158/1078-0432.Ccr-15-1136 (2016).

16. Ricciuti, B. et al. Impact of immune-related adverse events on survival in patients with advanced non-small cell lung cancer treated with nivolumab: longterm outcomes from a multi-institutional analysis. J Cancer Res Clin Oncol 145, 479-485, doi:10.1007/s00432-018-2805-3 (2019).

17. Matsuki, T. et al. Real-World, Long-Term Outcomes of Nivolumab Therapy for Recurrent or Metastatic Squamous Cell Carcinoma of the Head and Neck and Impact of the Magnitude of Best Overall Response: A Retrospective Multicenter Study of 88 Patients. Cancers (Basel) 12, doi:10.3390/cancers12113427 (2020).

18. Nishikawa, D. et al. Prognostic Markers in Head and Neck Cancer Patients Treated with Nivolumab. Cancers (Basel) 10, doi:10.3390/cancers10120466 (2018).

19. Simon, S. C. S. et al. Eosinophil accumulation predicts response to melanoma treatment with immune checkpoint inhibitors. Oncoimmunology 9 , 1727116, doi:10.1080/2162402x.2020.1727116 (2020).

20. Argiris, A., Li, Y. \& Forastiere, A. Prognostic factors and long-term survivorship in patients with recurrent or metastatic carcinoma of the head and neck. Cancer 101, 2222-2229, doi:10.1002/cncr.20640 (2004).

21. Chang, P. H. et al. Impact of the pretreatment Glasgow prognostic score on treatment tolerance, toxicities, and survival in patients with advanced head and neck cancer undergoing concurrent chemoradiotherapy. Head Neck 39, 1990-1996, doi:10.1002/hed.24853 (2017).

22. Niwa, K. et al. Multicentre, retrospective study of the efficacy and safety of nivolumab for recurrent and metastatic salivary gland carcinoma. Sci Rep 10, 16988, doi:10.1038/s41598-020-73965-6 (2020).

23. Ma, B. B. Y. et al. Antitumor Activity of Nivolumab in Recurrent and Metastatic Nasopharyngeal Carcinoma: An International, Multicenter Study of the Mayo Clinic Phase 2 Consortium (NCl-9742). J Clin Oncol 36, 1412-1418, doi:10.1200/jco.2017.77.0388 (2018).

24. Kokkali, S. et al. Nivolumab in patients with rare head and neck carcinomas: A single center's experience. Oral Oncol 101, 104359, doi:10.1016/j.oraloncology.2019.07.002 (2020). 
25. Saleh, K. et al. Response to salvage chemotherapy after progression on immune checkpoint inhibitors in patients with recurrent and/or metastatic squamous cell carcinoma of the head and neck. Eur J Cancer 121, 123-129, doi:10.1016/j.ejca.2019.08.026 (2019).

26. Pestana, R. C. et al. Response rates and survival to systemic therapy after immune checkpoint inhibitor failure in recurrent/metastatic head and neck squamous cell carcinoma. Oral Oncol 101, 104523, doi:10.1016/j.oraloncology.2019.104523 (2020).

27. Cabezas-Camarero, S. et al. Safety and Efficacy of Cetuximab-Based Salvage Chemotherapy After Checkpoint Inhibitors in Head and Neck Cancer. Oncologist, doi:10.1002/onco.13754 (2021).

28. Eisenhauer, E. A. et al. New response evaluation criteria in solid tumours: revised RECIST guideline (version 1.1). Eur J Cancer 45, 228-247, doi:10.1016/j.ejca.2008.10.026 (2009).

29. McMillan, D. C., Crozier, J. E., Canna, K., Angerson, W. J. \& McArdle, C. S. Evaluation of an inflammation-based prognostic score (GPS) in patients undergoing resection for colon and rectal cancer. Int J Colorectal Dis 22, 881-886, doi:10.1007/s00384-006-0259-6 (2007).

30. Michot, J. M. et al. Immune-related adverse events with immune checkpoint blockade: a comprehensive review. Eur J Cancer 54, 139-148, doi:10.1016/j.ejca.2015.11.016 (2016).

31. Common Terminology Criteria for Adverse Events (CTCAE) Version 5.0., https://ctep.cancer.gov/protocolDevelopment/electronic_applications/docs/CTCAE_v5_Quick_Reference_8.5x11.pdf\#search=\%22common\%20terminolog

32. Kanda, Y. Investigation of the freely available easy-to-use software 'EZR' for medical statistics. Bone Marrow Transplant 48, 452-458, doi:10.1038/bmt.2012.244 (2013).

\section{Tables}

Due to technical limitations, table 1 is only available as a download in the Supplemental Files section.

Table 2. Impact of clinical, hematological factors and prognostic score on clinical outcomes in recurrence or metastatic head and neck cancer patients treated with nivolumab. 


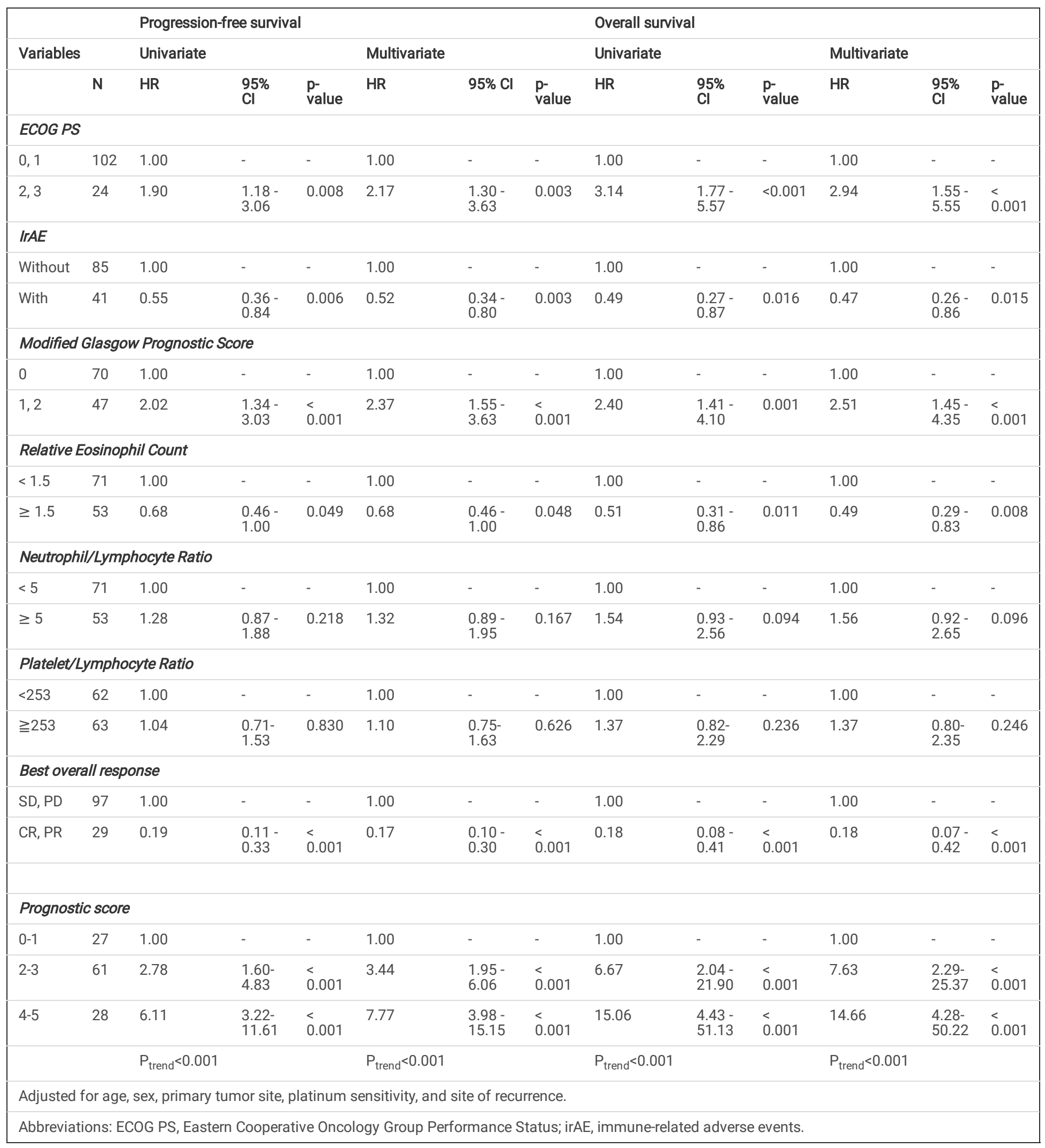

\section{Figures}


a

Progression-free survival

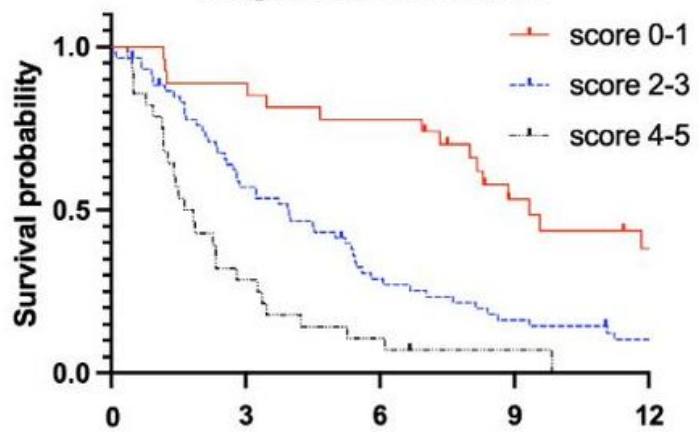

Number at risk

$\begin{array}{rrrrrr}\text { score } 0-1 & 27 & 25 & 22 & 13 & 8 \\ \text { score 2-3 } & 60 & 34 & 17 & 10 & 6 \\ \text { score 4-5 } & 28 & 9 & 4 & 2 & 1\end{array}$

b

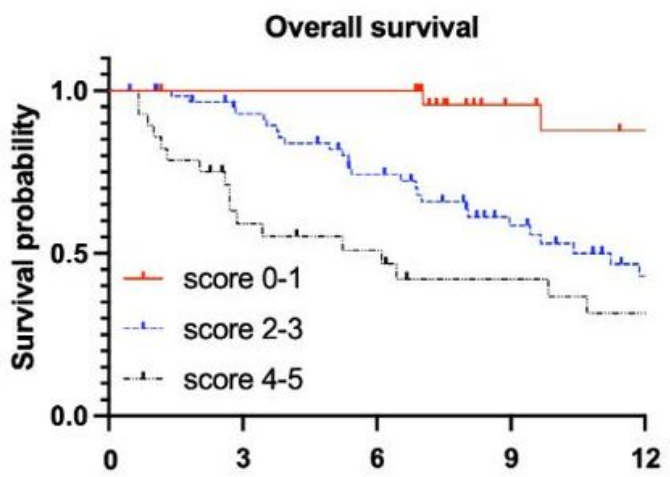

Number at risk

Survival (months)

$\begin{array}{cccccc}\text { score 0-1 } & 27 & 27 & 27 & 15 & 11 \\ \text { score 2-3 } & 61 & 52 & 39 & 23 & 13 \\ \text { score 4-5 } & 28 & 16 & 13 & 9 & 7\end{array}$

Figure 1

Kaplan-Meier curves of progression-free survival (PFS) and overall survival (OS) in patients with recurrent or metastatic head and neck cancer treated with nivolumab, stratified by prognostic score. Prognostic score is the sum of numbers from worse prognostic factors in clinico-hematological factors including without immune-related adverse events (irAEs), lower relative eosinophil count (REC), worse best overall response (BOR), higher Eastern Cooperative Oncology Group Performance Status (ECOG PS), and higher modified Glasgow Prognostic Score (mGPS). (a) Patients with a prognostic score of 4-5 (N=28) had significantly worse PFS than those with prognostic scores of $2-3(\mathrm{~N}=61)$ and $0-1(\mathrm{~N}=27)$ (1 year-PFS: $0.0 \%$ vs. 10.1\% [95\% Cl, 3.9-19.8] vs. 38.2\% [95\% $\mathrm{Cl}, 18.9$ $57.4], p<0.001)$. (b), Patients with a prognostic score of 4-5 ( $N=28)$ had significantly worse OS than those with prognostic scores of 2-3 ( $N=61)$ and $0-1(\mathrm{~N}=27)$ (1 year-OS: $26.3 \%$ [95\% Cl, 10.3-45.6] vs. $43.1 \%$ [95\% Cl, 27.4-57.9] vs. 87.7\% [95\% Cl, 56.9-97.0], p< 0.001).

\section{Supplementary Files}

This is a list of supplementary files associated with this preprint. Click to download.

- 0530Supplementary.pdf

- Table1.jpg 